\title{
Zika virus disease: a public health emergency
}

\author{
Jansen van Vuren P, Kemp A, Paweska J \\ Centre for Emerging and Zoonotic Diseases, National Institute for Communicable Diseases, Johannesburg, South Africa \\ Correspondence to: Prof J Paweska, e-mail: januszp@nicd.ac.za
}

The rise in microcephaly cases and neurological disorders reported in Brazil shortly after the emergence of Zika virus disease (ZVD) in 2015 prompted the World Health Organization (WHO) to declare ZVD as a Public Health Emergency of International Concern (PHEIC) on 1 February 2016. ${ }^{1}$ On 18 November 2016 the WHO Emergency Committee on Zika and microcephaly convened for their fifth meeting during which it was concluded that ZVD and associated neurological complications no longer represent a PHEIC, but the committee recognises that the unprecedented Zika virus (ZIKV) spread and its newly documented virulence remains a huge public health challenge requiring ongoing efforts. However, these efforts can be better addressed by sustained research programmes that are well resourced. ${ }^{2}$

Despite an apparent decrease in ZVD cases in recent months in affected regions of the world, the virus continues to expand its geographical range to areas where the mosquito vector is found. As of 17 November 2016 a total of 75 countries have reported autochthonous ZIKV transmission since 2007, of which 69 countries have been affected since 2015.

Currently there is no evidence of local transmission of ZIKV in South Africa, despite a small number of travel-associated introductions during 2016. ZIKV is mainly transmitted to humans by Aedes aegypti, the same mosquito vector responsible for transmission of other arboviruses of public health importance: dengue, chikungunya and yellow fever viruses. Aedes aegypti mosquitoes are found throughout South Africa but occur at highest density in the urban centres along the eastern coast. ${ }^{3}$ Unfortunately the areas with greatest abundance of the Zika mosquito vector include the more impoverished, high density housing areas, including those without access to piped water supply leading to container storage of water. Because water storage containers serve as suitable breeding sites for Aedes aegypti, ZIKV would most likely affect those already burdened by poverty if it became endemic in SA. About $80 \%$ of ZIKV infections are believed to be asymptomatic and clinical presentation during acute infections is not easily distinguishable from other infections. ${ }^{4}$ Laboratory testing is thus essential to make a diagnosis; however this is complicated by a transient viremia, resulting in a small window of opportunity to detect the virus, and extensive serological cross-reaction between viruses in the same family, making confirmation by antibody tests difficult. Due to these complications, a battery of laboratory tests, combined with consideration of clinical and epidemiological data, are often required to conclude suspected ZVD cases. These laboratory tests are highly specialised and expensive, making them practically inaccessible to those that will likely most need them if the virus became established in SA. As for any country affected by such an emergency, public health interventions would need to be activated at government level and would most likely require the approval of emergency funds for a proper public health response. The US Centers for Disease Control and Prevention had set aside $\$ 222$ million to respond to the outbreak, of which \$194 million had already been spent by the end of August 2016. This highlights the scale of the financial resources that would be required to mount a proper public health response. Funding is however a universal problem, with the WHO's emergency fund having a balance of only $\$ 31.5$ million to respond to a variety of ongoing health threats. ${ }^{5}$

How did South Africa respond? Shortly after the announcement of Zika and associated complications being a PHEIC, the South African National Department of Health, Environmental Health Directorate, issued an alert to intensify surveillance and screening for ZIKV at points of entry. This includes a number of steps: 1) strengthening of protocols to spray arriving aircraft with insecticide and monitor airports for the presence of mosquitoes; 2) assessment of sea ports and harbours to a) identify possible vector breeding areas; $b$ ) ensure that steps have been taken to minimise insect breeding, and c) to inspect cargo for presence of mosquito vectors; 3 ) continued thermal scanning of all travellers at ports of entry; 4) referral of travellers at points of entry with compatible symptoms to a healthcare facility for further management; 5) increased monitoring of imported used tyre casings and 6) increased health education and awareness about ZIKV disease for travellers.

In addition the National Department of Health, the Chief Directorate: Communicable Diseases recently established a Zika and yellow fever technical working group. The working group, formally established on 5 September 2016, consists of experts in relevant fields including epidemiologists, clinicians, laboratory diagnosticians and entomologists. The functions of the working group are to: 1) provide technical advice on Zika and yellow fever response; 2 ) support the development and implementation of the Zika and yellow fever preparedness plans; 3 ) provide advice 
on prevention of introduction and surveillance for vectors; 4) advise on case management and public health response; 5) provide advice on risk communication i.e. health education and public communications; 6) advise on technical, financial and human resources required; 7) review knowledge on diagnostics and vaccines and advise on required research programmes.

The number of countries reporting microcephaly or central nervous system abnormalities associated with ZIKV infection continues to expand into Africa; Cabo Verde reporting nine ZVD cases. ${ }^{6}$ This continued geographic expansion raises the concern that the end of the PHEIC was declared prematurely by the WHO Director-General. Economic losses in Latin America attributable to the Zika epidemic are estimated at $\$ 3.5$ billion, but this does not yet take into account the massive future burden on the healthcare system and economy of caring for disabled children as a result of Zika virus infection. ${ }^{5}$ In addition to the direct effects of the virus on pregnant women and their unborn children, some have suggested that it might also indirectly result in a so-called Zika generation. ${ }^{5}$ This generation might end up living in fear of conception, which would eventually result in decreased birth rates, and subsequently lowered productivity and economic growth. It remains to be seen how Africa, where the virus is thought to be endemic, will be affected directly by the ongoing epidemic. It is not known whether microcephaly and other neurological complications might always have been associated with Zika virus infection on the African continent and that it was only misdiagnosed or underreported, or whether genetic changes in the virus resulted in its new propensity to cause neurological disorders and its better fitness to spread through Asia to Latin America. It is not known what effect background immunity to the African Zika virus lineage will have in the event of the Asian lineage becoming endemic. Will immunity to African lineage Zika virus protect individuals from re-infection with Asian lineage virus, or might it result in immune enhancement leading to even more severe complications? Regardless of the endemicity of Zika virus on the African continent, introduction of the Asian lineage could have severe implications. The majority of African countries' public health systems are under resourced and underprepared to deal with emergence or re-emergence of infectious diseases, a fact so painfully highlighted by the largest ever Ebola virus disease outbreak in West Africa recently. The effects on socio-economic wellbeing, political stability, tourism and trade could be severe on a continent already plagued by so many health and socio-economic challenges.

\section{References}

1. World Health Organization. WHO statement on the first meeting of the International Health Regulations (2005) (IHR 2005) Emergency Committee on Zika virus and observed increase in neurological disorders and neonatal malformations. 1 February 2016. http://www.who.int/mediacentre/news/ statements/2016/1st-emergency-committee-zika/en/

2. World Health Organization. WHO statement on the fifth meeting of the International Health Regulations (2005) (IHR 2005) Emergency Committee on Zika virus and observed increase in neurological disorders and neonatal malformations. 18 November 2016. http://www.who.int/mediacentre/news/ statements/2016/zika-fifth-ec/en/

3. Kemp A, Jupp PG. Potential for dengue in South Africa: mosquito ecology with particular reference to Aedes aegypti. J Am Mos Contr Ass., 1991;7(4):574-83.

4. Simpson DI. Zika virus infection in man. Trans R Soc Trop Med Hyg 1964;58(4):335-338.

5. Gostin LO, Hodge Jr JG. Zika virus and global health security. The Lancet, 2016;16:1099-1100.

6. World Health Organization. Zika situation report. 17 November 2016. http:// www.who.int/emergencies/zika-virus/situation-report/17-november-2016/en/ 